\title{
Bilateral use of Active Middle Ear Implants: Speech Discrimination Results in Noise \\ ${ }^{1}$ A.Wolf-Magele, ${ }^{2}$ V.Koci, ${ }^{1} \mathrm{~J} . S c h n a b l,{ }^{2}$ P.Zorowka, ${ }^{3} \mathrm{H}$. Riechelmann, ${ }^{1} \mathrm{GM}$ Sprinzl \\ ${ }^{1}$ Department of Otorhinolaryngology, Karl Landsteiner Private University St. Poelten, University Clinic St.Poelten, Austria \\ ${ }^{2}$ Department for Hearing, Speech and Voice Disorder, ${ }^{3}$ Department of Otorhinolaryngology - University Clinic Innsbruck, Austria
}

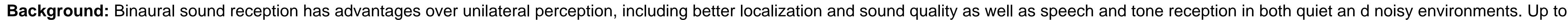

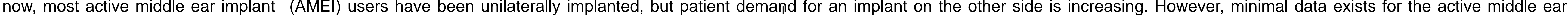

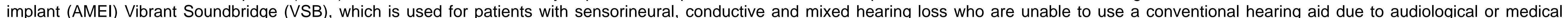

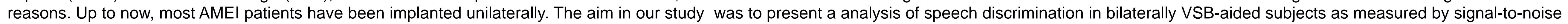
ratios for $50 \%$ correct understanding of words in sentences.

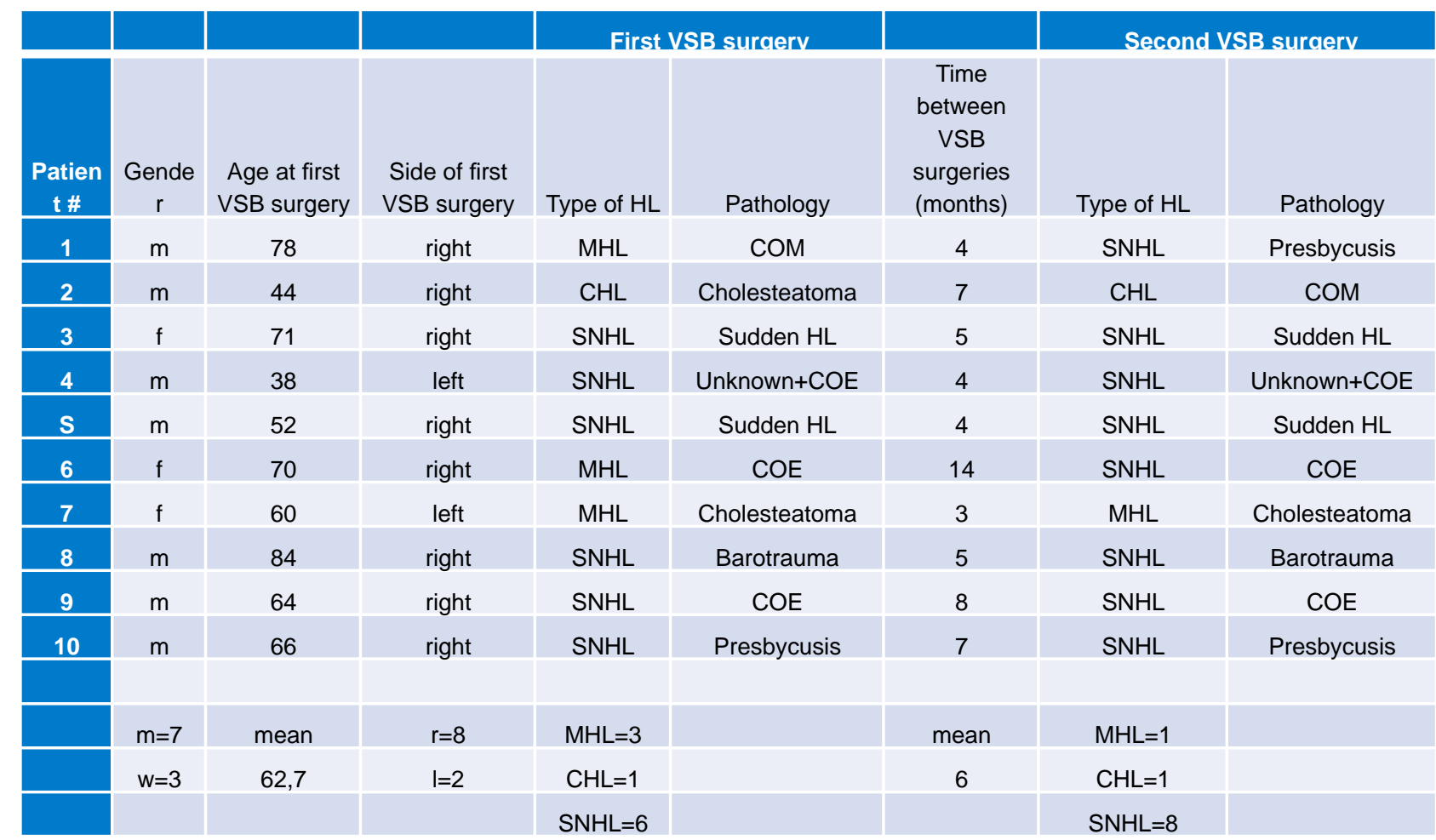

Table 1: Subjects' demographic overview (COM: chronic otitis media; COE: chronic otitis externa; MHL: mixed hearing loss; CHL: conductive hearing loss; Presbycusis: no satisfactory amplification with conventional hearing aid)

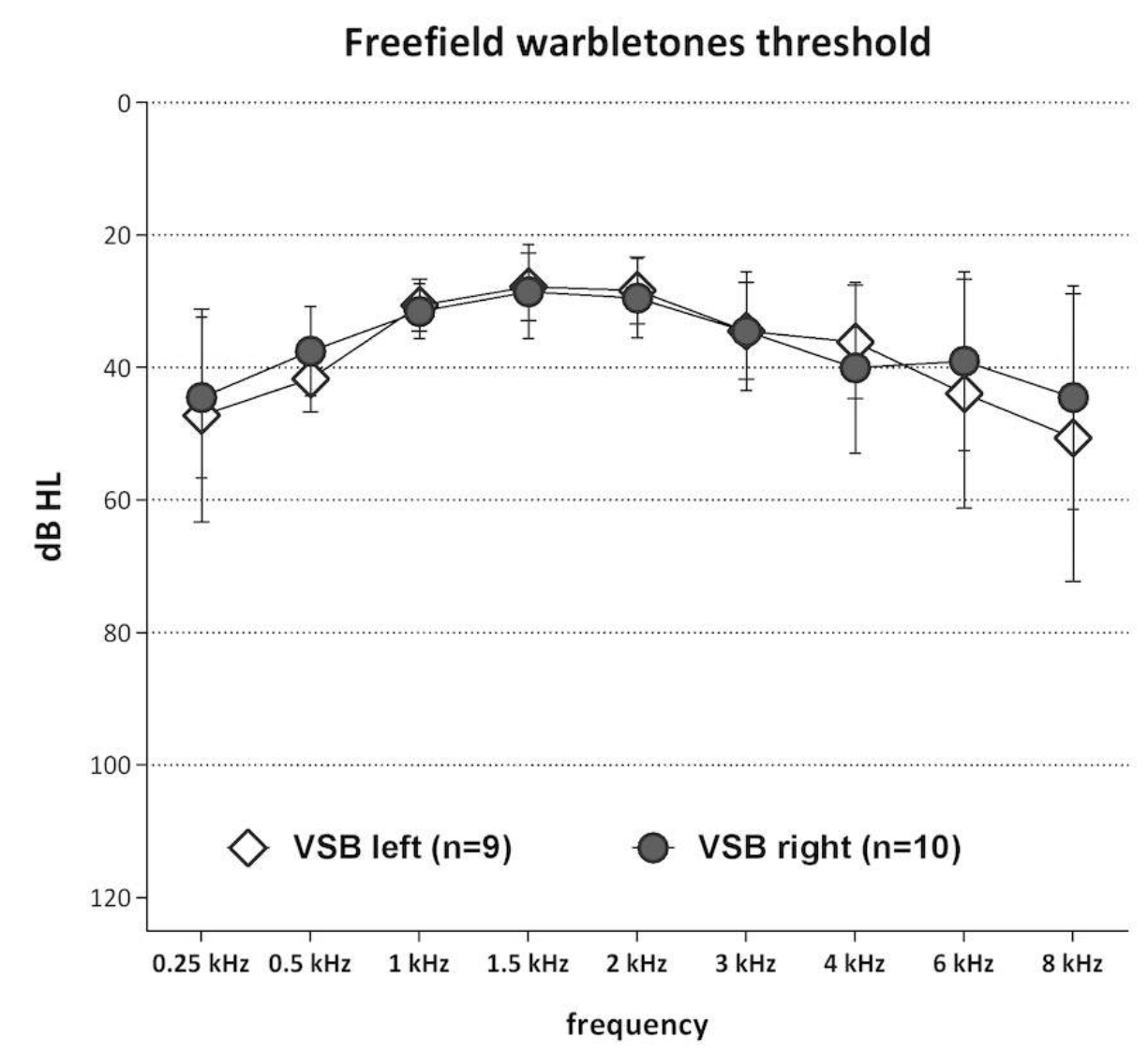

Figure 1: Mean free field post-op warble tone threshold for both implanted ears: error bars reflect \pm standard deviation.

Material \& Methods: Ten bilaterally-AMEl implanted native German-speaking adults were included in the study. The Oldenburg sentence test was used to measure speech reception thresholds in noise. The subject's signal-to-noise ratio (SNR) at a speech reception score of $50 \%$ was calculated for different noise conditions. SRT was measured as a function of noise condition (nc) and listening condition (Ic) - for example, SRT (Ic,nc), with nc from SONO, SON-90, or SON90 and Ic from left, right or both. For each noise condition, the squelch effect and the binaural summation effect was calculated.

$$
\text { Summation effect }
$$
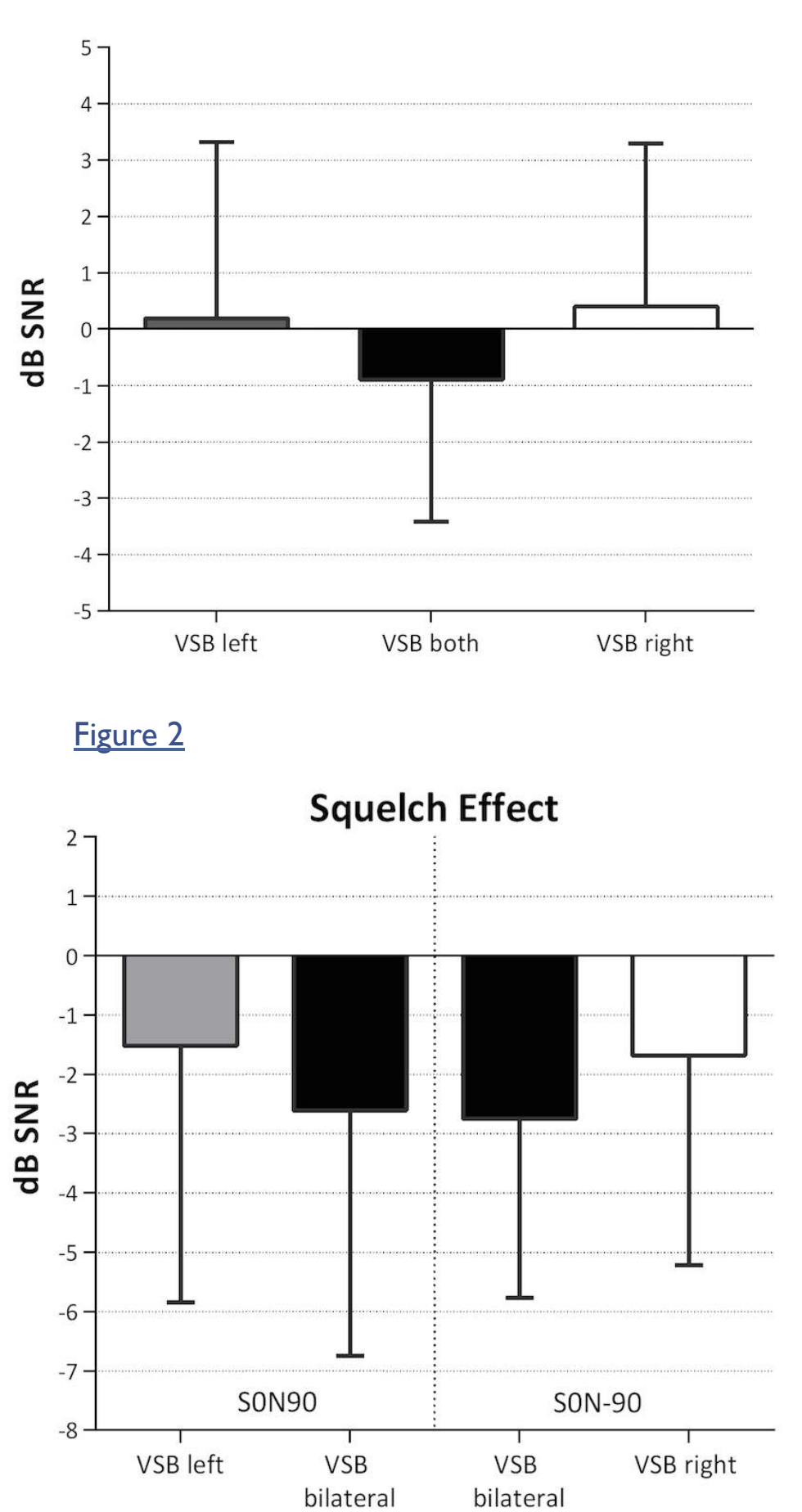

Figure 4

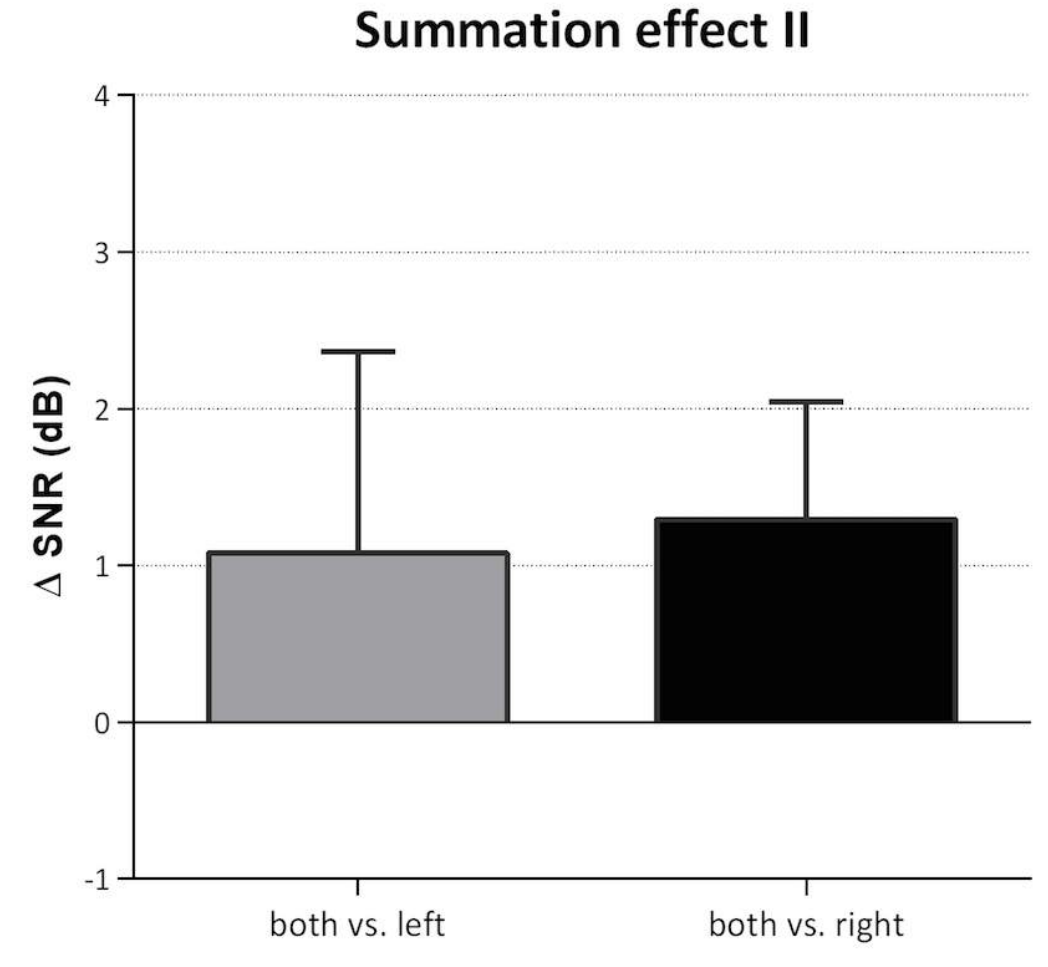

Figure 3

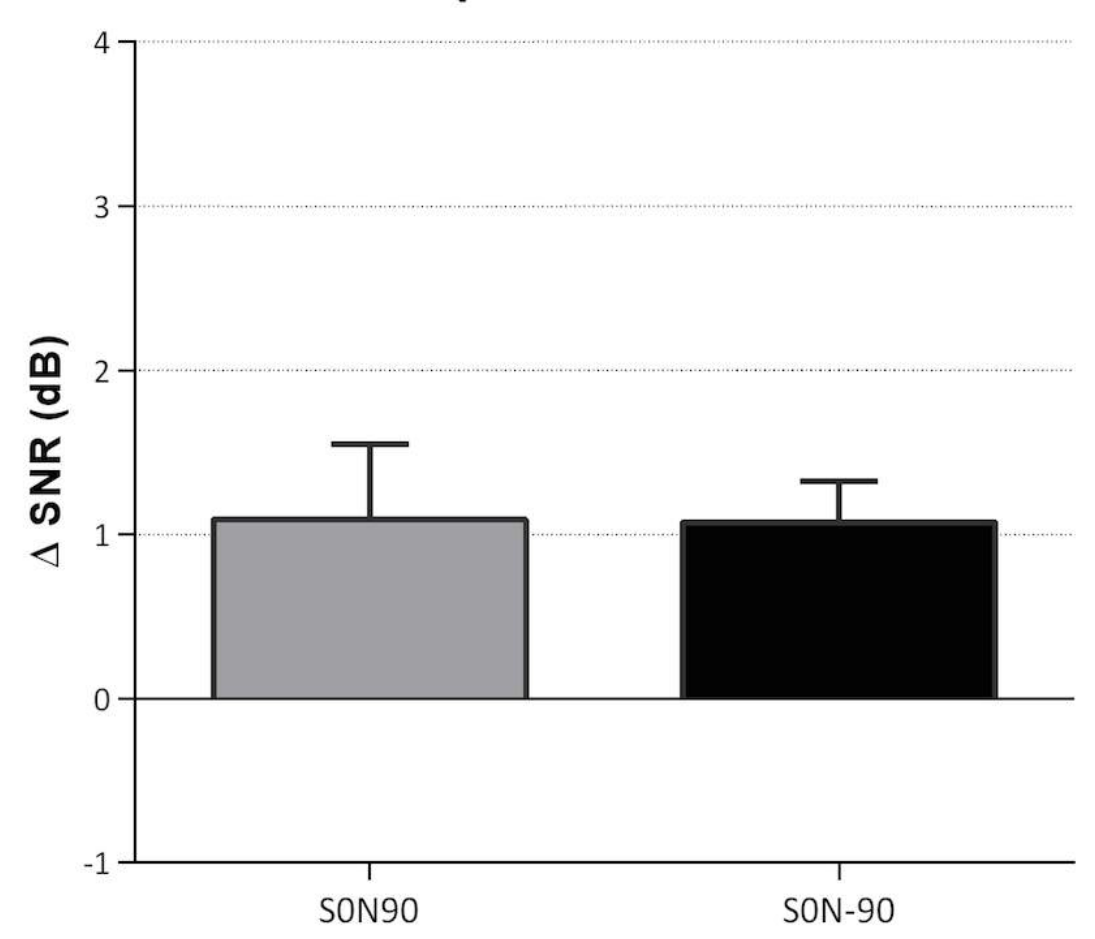
Table 2: Mean differences in bone-, and air-conduction thresholds

Results: Patients in this study demonstrated improvement with bilateral AMEls compared to right or left AMEI only in all three tested listening conditions. Statistical significance was found in the SONO condition to favor usage of bilateral AMI versus either the right or left side only.

Discussion: The benefits of binaural hearing are well known, also in normal-hearing individuals. In the future every bilateral implantation should be a part of the clinical routine. Bilateral implantation can help to reduce problems in background noise and restore directional hearing. AMEls such as the VSB help patients with high-frequency hearing loss, occlusion or distortion problems, as well as other medical problems preventing permanent hearing aid usage. Bilateral VSBs can help to reduce problems in background noise and restore directional hearing.

2: Mean SNR in listening condition SONO. Error bars reflect standard deviation.

(the right SONO) R leftright - SNR both in listening condition

4: Mean SNR with noise from the righ

(SON9O) and noise from the left side (SO right side reflect standard deviation.

Figure 5: Mean Squelch effect with noise from the right side (SON90) and noise from the left side (SON-90). Error bars reflect standard deviation 